scientific curiosity; they may also have practical consequences. Research has confirmed the prediction (originally made on theoretical grounds) that a high tendency to see objects in their real sizes irrespective of distance should be of advantage to the drivers of motor vehicles. The amount of this tendency may be altered by the action of drugs and by the presence of mist and fog, and this change may affect driving. One case has been reported of its change in a neurotic condition.

The psychology of vision can no longer be treated as if vision were a function of the eye alone. The foundations of a psychology of vision were firmly laid by the work of Helmholtz and his contemporaries, who made a scientific study of the sensory physiology of the eye. The most fruitful field of research at present is in those wider problems of visual perception in which the eye and the higher centres co-operate.

\section{Physiology of the Plant Cell}

P ROF. W. STILES, in his presidential address to Section K (Botany), defines the general physiology of the plant cell as those vital activities of plants which are manifested by every living cell, and are thus distinguished from those special processes such as photosynthesis, which are restricted to certain specialized organs. These general activities of living matter are respiration and the absorption and excretion of water and dissolved substances.

The usually accepted meaning of respiration is that it provides the energy for plant movements and the building up from the products of photosynthesis of substances of higher energy content than these products. Yet as respiration is a constant property of living matter, even when there is no movement and no formation of fresh material it may be questioned whether this current view of respiration is complete. As regards the synthesis of proteins and other complex substances, while there is evidence of the linkage of anabolic processes with the breaking down of carbohydrate, this anabolism only concerns the re-formation of carbohydrates, and no evidence has yet been obtained which sheds any light on the way in which energy released in respiration is transferred to the processes involved in the synthesis of proteins and other substances of higher complexity than those forming the respiratory substrate.

The absorption of water and dissolved substances by plant cells-was formerly assumed to be a simple process of diffusion through cell mem. branes, but during the present century experimental work has shown that while the absorption of non-electrolytes may sometimes take place in this way, the absorption of electrolytes is a much more complex process, for the unequal absorption of the two ions of a salt, and the entrance of the ions of a salt against their own concentration gradients are very general phenomena. Theories based on adsorption and interchange of ions have been put forward to account for these observed facts, but while such processes probably operate, it is doubtful whether they afford a complete explanation of the phenomena.

The connexion between respiration and the salt relations of tissues was pointed out in 1927, and since then further evidence has been forthcoming which has emphasized the connexion between respiration and the diffusion of salts into plant cells against the concentration gradient. The energy required for the entrance of salt thus is presumably provided by respiration, and theories have been enunciated which regard the absorption of ions as a continuous interchange of the former with the hydrogen and bicarbonate ions continuously produced within the cell as a consequence of respiration. While this interchange may be a factor in salt accumulation, there is evidence that the connexion between respiration and accumulation is generally much more indirect, and that the failure of cells to accumulate salt when respiration is lowered through deprivation of the cells of an adequate oxygen supply is related to their generally lowered vitality under this condition.

It is emphasized that salt accumulation, like respiration itself, is a vital process dependent on the protoplasm, while there is some evidence that in the absorption of water by the plant cell, there is in some, though not all, cases an active secretion of water. It is thus clear that a further analysis of the protoplasmic system is necessary for a better understanding of general cell physiology.

The general physiology of the cell is not only of fundamental importance for plant physiology in general, but it is of similar importance for all ecological investigation which is not merely descriptive, while it also impinges on the important fields of mycology and cyto-genetics. Cell physiology is the scientific basis of many important plant industries, including those of food preservation and storage.

\section{Administration of Public Education}

IN his presidential address to Section $\mathrm{L}$ (Education) Mr. John Sargent discusses the part which administration, and local administration in particular, ought to play as a medium through which the basic principles of educational science, as revealed either by a priori reasoning or as the 